\title{
QUASI-ISOMETRIC EXTENSIONS OF QUASISYMMETRIC MAPPINGS OF THE REAL LINE COMPATIBLE WITH COMPOSITION
}

\author{
Zair Ibragimov \\ California State University, Department of Mathematics \\ Fullerton, CA 92834, U.S.A.; zibragimov@fullerton.edu
}

\begin{abstract}
We show that it is possible to extend, in a homomorphic fashion, each quasisymmetric homeomorphism of the real line to a quasi-isometry of the upper-half plane. Epstein and Markovic have recently shown that a homomorphic extension to quasiconformal homeomorphisms of the upper-half plane is not possible.
\end{abstract}

\section{Introduction}

The quasisymmetric mappings of the real line $\mathbf{R}$ were first considered in the work of Beurling and Ahlfors [3], where they appeared as the boundary mappings induced by the quasiconformal self-mappings of the upper-half plane $\mathbf{H}^{2}$. In particular, Beurling and Ahlfors constructed an extension operator, which extends each quasisymmetric mapping of $\mathbf{R}$ to a quasiconformal self-mapping of $\mathbf{H}^{2}$. In general, it is desirable to have an extension operator with some additional properties. Tukia [14] has constructed an extension operator compatible with a Möbius group while Douady and Earle [5] have constructed a continuous, conformally natural extension operator which extends each quasisymmetric self-mapping of the circle $\mathbf{S}^{1}$ to a quasiconformal diffeomorphism of the disk $\mathbf{D}^{2}$. Because of its potential applications in geometric function theory as well as in low dimensional geometry and topology, an extension operator compatible with a composition is one of the most desirable ones. Both the Beurling-Ahlfors and the Douady-Earle extensions lack the compatibility property. The Dream Problem of Sullivan asks if there exists an operator compatible with a composition, which extends each quasisymmetric homeomorphism of the circle $\mathbf{S}^{1}$ to a quasiconformal homeomorphism of the disk $\mathbf{D}^{2}$. Recently, Epstein and Markovic have shown that no such operator exists [8].

Although the Dream Problem has been solved, there are other (weaker) versions of it that are still open. Recall that the quasiconformal homeomorphisms of $\mathbf{H}^{2}$ onto itself are quasi-isometries of $\mathbf{H}^{2}$ in the hyperbolic metric (see, for instance, [7, Theorem 13.6]). Also, each quasi-isometry of $\mathbf{H}^{2}$ induces a quasisymmetric self-mapping of $\overline{\mathbf{R}}=\mathbf{R} \cup\{\infty\}$. The origin of this result goes back to the work of Efremovich and Tihomirova [6]. Here the set $\overline{\mathbf{R}}$ is considered as the boundary at infinity of the hyperbolic space $\mathbf{H}^{2}$ endowed with the chordal metric, which is a visual metric, and the induced map need not fix $\infty$. Conversely, each quasisymmetric self-mapping of $\overline{\mathbf{R}}$ can be extended to a quasi-isometry of $\mathbf{H}^{2}$. (In fact, all the quasiconformal

doi:10.5186/aasfm.2010.3513

2000 Mathematics Subject Classification: Primary 30C62, 37E10, 37E30.

Key words: Quasiconformal mappings, quasisymmetric mappings, quasi-isometry, convergence groups, hyperbolic space. 
extension operators mentioned above yield extensions that are biLipschitz in the hyperbolic metric). Hence it is natural to ask if there exists a quasi-isometric extension operator compatible with a composition. We call it the quasi-isometric version of the Dream Problem. One can also pose a convergence group version of the Dream Problem: find an extension operator which extends each convergence group on $\mathbf{S}^{1}$ to a convergence group on $\overline{\mathbf{D}^{2}}$. A positive solution of this problem in combination with a result of Martin and Tukia that convergence groups on $\overline{\mathbf{D}^{2}}$ are topologically conjugate to Fuchsian groups (see, [12, Theorem 4.4]) would give another solution of the Fuchsian Group Conjecture. Recall that the Fuchsian Group Conjecture contains the Nielsen Realization Problem as well as the Seifert Fibered Space Conjecture and was completely solved in the early 1990's independently by Gabai [9] and Casson and Jungreis [4]. (See also [15] and [11]). Norton and Sullivan have considered other versions of the Dream Problem (see [13, p. 59-61]). We remark that the Dream Problem as well as the two versions of it mentioned above are still open in higher dimensions (see also [8, p. 522]) .

It appears that the extension problem for the quasisymmetric mappings is more natural if one considers the quasisymmetric mappings as the induced boundary mappings of the quasi-isometries rather than those of the quasiconformal mappings. In this paper we show that the quasi-isometric version of the Dream Problem has a positive solution. More precisely, we show that the operator $\mathscr{H}: \operatorname{Homeo}(\mathbf{R}) \rightarrow$ Homeo $\left(\mathbf{H}^{2}\right)$, defined in Section 3, satisfies the following properties: (A) $\mathscr{H}\left(\phi_{1} \circ \phi_{2}\right)=$ $\mathscr{H}\left(\phi_{1}\right) \circ \mathscr{H}\left(\phi_{2}\right)$ for all $\phi_{1}, \phi_{2} \in \operatorname{Homeo}(\mathbf{R}) ;(\mathrm{B}) \mathscr{H}(a t+b)=a z+b$ for all $a>0$ and $b \in \mathbf{R} ;(\mathrm{C})$ if $\phi$ is $K$-quasisymmetric, then $\mathscr{H}(\phi)$ is $(\lambda, k)$-quasi-isometry, where $\lambda$ and $k$ depend only on $K$ (Theorem 3.1). The operator $\mathscr{H}$ was already known to Beurling and Ahlfors, who considered a modified version of it in order to achieve quasiconformal extensions at the cost of properties (A) and (B) (see [3, p. 135]). The operator $\mathscr{H}$ was also considered by Norton and Sullivan, who observed that it solves the Dream Problem in the restricted category of biLipschitz homeomorphisms of $\mathbf{R}$ (see $[13$, p. 59$]$ ). One can easily see that the extension $\mathscr{H}(\phi)$ of the quasisymmetric map $\phi(t)=t^{3}$ is not quasiconformal and, in particular, it is not biLipschitz in the hyperbolic metric. The reason why property $(\mathrm{C})$ holds is the fact that $\mathscr{H}$ commutes with all the affine Möbius transformations, i.e., $\mathscr{H}(a \phi+b)=a \mathscr{H}(\phi)+b$ for all $\phi \in \operatorname{Homeo}(\mathbf{R}), b \in \mathbf{R}$ and $a>0$. In addition, the operator $\mathscr{H}$ gives rise to a new extension operator $\mathscr{H}_{o}: \mathrm{Homeo}_{+}\left(\mathbf{S}^{1}\right) \rightarrow \operatorname{Homeo}\left(\mathbf{D}^{2}\right)$ (suggested to us by Vlad Markovic), which is compatible with composition (see (3.2)). It would be interesting to study the operator $\mathscr{H}_{o}$ in connection with the convergence group version of the Dream Problem. We thank Vlad Markovic for many valuable suggestions, which have significantly improved the presentation.

Finally, Pekka Tukia has pointed out to us that our extension is slightly more regular than just quasi-isometry, namely, if $\phi$ is $K$-quasisymmetric, then $\mathscr{H}(\phi)$ has a modulus of continuity with respect to the hyperbolic metric depending only on $K$ (Lemma 3.2). He also pointed out that the composition property of $\mathscr{H}$ implies that the Beurling-Ahlfors extension is a "rough" homomorphism (Corollary 4.2). We want to thank Pekka Tukia for his helpful suggestions and comments as well as for encouraging us to write Section 4. 


\section{Preliminaries}

The real and complex numbers are denoted by $\mathbf{R}$ and $\mathbf{C}$, respectively. Let $\mathbf{S}^{1}=$ $\{z \in \mathbf{C}:|z|=1\}, \mathbf{D}^{2}=\{z \in \mathbf{C}:|z|<1\}$ and $\mathbf{H}^{2}=\{z \in \mathbf{C}: \operatorname{Im}[z]>0\}$. If $X$ is one of the sets $\mathbf{R}, \mathbf{S}^{1}, \mathbf{H}^{2}$ or $\mathbf{D}^{2}$, we let $\operatorname{Homeo}(X)$ denote the family of selfhomeomorphisms of $X$. The set $\mathbf{H}^{2}$ is endowed with the hyperbolic metric $h$, given by

$$
h(z, w)=\inf _{\gamma} \int_{a}^{b} \frac{\left|\gamma^{\prime}(t)\right|}{\operatorname{Im}[\gamma(t)]} d t,
$$

where the infimum is taken over all piecewise continuously differentiable curves $\gamma:[a, b] \rightarrow \mathbf{H}^{2}$ joining $z$ and $w$. The boundary at infinity of the hyperbolic space $\left(\mathbf{H}^{2}, h\right)$ is identified with the one-point compactification $\overline{\mathbf{R}}=\mathbf{R} \cup\{\infty\}$ of $\mathbf{R}$ and is denoted by $\partial \mathbf{H}^{2}$. The hyperbolic metric $h$ can also be defined as

$$
h(z, w)=\log \frac{\left|z-w^{\star}\right|\left|w-z^{\star}\right|}{\left|z-z^{\star}\right|\left|w-w^{\star}\right|},
$$

where $\left\{z^{\star}, w^{\star}\right\}=S(z, w) \cap \partial \mathbf{H}^{2}$ so that the points $z^{\star}, z, w, w^{\star}$ lie on $S(z, w)$ in this order and $S(z, w)$ is the unique circle in $\overline{\mathbf{C}}=\mathbf{C} \cup\{\infty\}$ passing through $z$ and $w$ and orthogonal to $\partial \mathbf{H}^{2}$ (see [2, p. 133]). Next, for each $z, w \in \mathbf{H}^{2}$ with $z=x+i y$ and $w=u+i v$, we put

$$
\rho(z, w)=\max _{s \in A, t \in B}|s-t|,
$$

where $A=\{x-y, x+y\}$ and $B=\{u-v, u+v\}$. The following observation as well as the next lemma will be used in the proof of our main theorem: there exist $s, s^{\prime} \in A$ and $t, t^{\prime} \in B$ such that

$$
\frac{\rho(z, w)}{2 \operatorname{Im}[z]}=\frac{|s-t|}{\left|s-s^{\prime}\right|} \geq \frac{1}{2} \quad \text { and } \quad \frac{\rho(z, w)}{2 \operatorname{Im}[w]}=\frac{|t-s|}{\left|t-t^{\prime}\right|} \geq \frac{1}{2} .
$$

Lemma 2.1. For all $z, w \in \mathbf{H}^{2}$ we have

$$
0 \leq \log \frac{\rho^{2}(z, w)}{\operatorname{Im}[z] \operatorname{Im}[w]}-h(z, w) \leq \log 4
$$

The inequalities are sharp.

Proof. Let $z=x+i y$ and $w=u+i v$. Assume first that $x=u$. Then $\rho(z, w)=$ $y+v$ and $(2.3)$ holds as it is equivalent to $\max \{y, v\} \leq y+v \leq 2 \max \{y, v\}$. The latter also shows the sharpness of lower and upper bounds in (2.3) as $\min \{y, v\} \rightarrow 0$ and as $y \rightarrow v$, respectively. Assume now that $x \neq u$. Let $\gamma$ be the unique hyperbolic geodesic joining $z$ and $w$. Since (2.3) is invariant under a preliminary transformations of the form $z \mapsto k z, z \mapsto z+a$, and $z \mapsto b-\bar{z}$, we can further assume that $\gamma=\mathbf{H}^{2} \cap \mathbf{S}^{1}$ and $x<u$. Then

$$
h(z, w)=\log \left(\frac{|z-1|}{|z+1|} \cdot \frac{|w+1|}{|w-1|}\right)=\log \left(\frac{\sqrt{1-x}}{\sqrt{1+x}} \cdot \frac{\sqrt{1+u}}{\sqrt{1-u}}\right) .
$$

On the other hand, the condition $x<u$ implies that $\rho(z, w)=|x-y-u-v|=$ $u+v-x+y$. Hence 


$$
\begin{aligned}
\log \frac{\rho^{2}(z, w)}{\operatorname{Im}[z] \operatorname{Im}[w]}-h(z, w) & =\log \frac{(u+v-x+y)^{2}}{\sqrt{1-x^{2}} \sqrt{1-u^{2}}} \frac{\sqrt{1+x}}{\sqrt{1-x}} \frac{\sqrt{1-u}}{\sqrt{1+u}} \\
& =\log \frac{(u+v-x+y)^{2}}{(1-x)(1+u)} .
\end{aligned}
$$

We need to show that

$$
1 \leq f(x, u) \leq 4, \quad \text { where } \quad f(x, u)=\frac{(u+v-x+y)^{2}}{(1-x)(1+u)} .
$$

Since $f(x, u)=f(-u,-x)$, we can assume that $-1<x \leq 0$. Then $y-x \geq 1$ and hence

$$
f(x, u)=\frac{(u+v-x+y)^{2}}{(1-x)(1+u)} \geq \frac{(u+v+1)^{2}}{(1-x)(1+u)}=\frac{2+2 v}{1-x} \geq 1 .
$$

To show the upper bound, we consider two cases. First, if $0 \leq u \leq 1$, then one can easily check that for each fixed $x$, the function $f(x, u)$ is a decreasing function of $u$. Hence

$$
f(x, u) \leq f(x, 0)=\frac{(1-x+y)^{2}}{1-x}=2+2 y \leq 4 .
$$

Second, if $x \leq u \leq 0$, then for each fixed $u$, the function $f(x, u)$ is an increasing function of $x$. Hence

$$
f(x, u) \leq f(u, u)=\frac{4 v^{2}}{1-u^{2}}=4
$$

The proof is complete.

The notion of quasisymmetry is due to Beurling and Ahlfors ([3]). An increasing homeomorphism $\phi$ of $\mathbf{R}$ onto itself is called $K$-quasisymmetric if

$$
\frac{1}{K} \leq \frac{\phi(x+t)-\phi(x)}{\phi(x)-\phi(x-t)} \leq K
$$

holds for all $x, t \in \mathbf{R}$ with $t \neq 0$. The quasisymmetric mappings in general metric spaces have been introduced and studied by Tukia and Väisälä [17]. A homeomorphism $f$ between the metric spaces $(X,|-|)$ and $(Y,|-|)$ is called quasisymmetric if there exists a homeomorphism $\eta:[0,+\infty) \rightarrow[0,+\infty)$ such that

$$
\frac{|f(a)-f(b)|}{|f(a)-f(c)|} \leq \eta\left(\frac{|a-b|}{|a-c|}\right)
$$

holds for all distinct $a, b, x \in X$. We also say that $f$ is $\eta$-quasisymmetric. If $f$ is $\eta$-quasisymmetric with

$$
\eta(t)= \begin{cases}\lambda t^{1 / \alpha} & \text { if } 0 \leq t \leq 1 \\ \lambda t^{\alpha} & \text { if } t \geq 1\end{cases}
$$

for some $\lambda \geq 1$ and $\alpha \geq 1$, then we say that $f$ is $(\lambda, \alpha)$-quasisymmetric. If $f$ is $(\lambda, \alpha)$ quasisymmetric, then one can easily observe that $f^{-1}$ is $\left(\lambda^{\alpha}, \alpha\right)$-quasisymmetric. Recall that for homeomorphisms of $\mathbf{R}$ onto itself the conditions (2.4) and (2.5) are equivalent and that each $\eta$-quasisymmetric map of $\mathbf{R}$ is $(\lambda, \alpha)$-quasisymmetric for some $\lambda \geq 1$ and $\alpha \geq 1$ depending only on $\eta$ (see [17, Theorem 10.3 and Corollary 3.12$])$. 
A map $f: X \rightarrow Y$ is called a quasi-isometry if there exist constants $\lambda \geq 1$ and $k \geq 0$ such that

$$
\lambda^{-1}|a-b|-k \leq|f(a)-f(b)| \leq \lambda|a-b|+k
$$

for all $a, b \in X$ and if

$$
\inf _{x \in X}|f(x)-y| \leq k \quad \text { for all } \quad y \in Y .
$$

We also say that $f$ is $(\lambda, k)$-quasi-isometry (see [18]). Note that quasi-isometries need not be continuous. A homeomorphism $f: X \rightarrow Y$ is said to be biuniform if there exist two homeomorphisms $\eta$ and $\eta^{\prime}$ of $[0, \infty)$ such that

$$
\eta^{\prime}(|x-y|) \leq|f(x)-f(y)| \leq \eta(|x-y|)
$$

for all $x, y \in X$ (see [16]). We also say that $f$ is $\left(\eta, \eta^{\prime}\right)$-biuniform.

In this paper we only consider the homeomorphisms of $\mathbf{H}^{2}$ in connection with quasi-isometries. Hence we say that a map $f \in \operatorname{Homeo}\left(\mathbf{H}^{2}\right)$ is quasi-isometry if there exists $k \geq 0$ and $\alpha \geq 1$ such that

$$
\frac{1}{\alpha} h(z, w)-k \leq h(f(z), f(w)) \leq \alpha h(z, w)+k
$$

for all $z, w \in \mathbf{H}^{2}$. If $f$ is a $\left(\eta, \eta^{\prime}\right)$-biuniform homeomorphism of the hyperbolic space $\mathbf{H}^{2}$, then for each $k>0$ the map $f$ is $(\lambda, k)$-quasi-isometric, where $\lambda$ depends only on $k, \eta$ and $\eta^{\prime}$ (see, [16, Lemma 3C]).

The notion of convergence groups is due to Gehring and Martin ([10]). Tukia [16] studied the convergence groups in the settings of compact metric spaces. Let $X=\mathbf{S}^{1}$ or $\overline{\mathbf{D}^{2}}$. A group $G \subset \operatorname{Homeo}(X)$ is called a convergence group if for each sequence of distinct elements of $G$ there exists $s, t \in X$ and a subsequence $\left\{\phi_{k}\right\}$ such that $\left\{\phi_{k}\right\} \rightarrow s$ and $\left\{\phi_{k}^{-1}\right\} \rightarrow t$ uniformly on compact subsets of $X \backslash\{t\}$ and $X \backslash\{s\}$, respectively. Examples of convergence groups are Möbius groups and their topological conjugates. The convergence groups on $\mathbf{R}$ are, by definition, the convergence groups on $\overline{\mathbf{R}}$ whose elements fix $\infty$.

\section{The extension operator $\mathscr{H}$}

We define an extension operator $\mathscr{H}$ on $\operatorname{Homeo}(\mathbf{R})$ as follows. Given $\phi \in$ Homeo $(\mathbf{R})$, let

$$
\mathscr{H}(\phi)(z)=\mathscr{H}(\phi)(x+i y)=\alpha(x, y)+i \beta(x, y)
$$

where

$$
\alpha(x, y)=\frac{\phi(x+y)+\phi(x-y)}{2} \quad \text { and } \quad \beta(x, y)=\frac{|\phi(x+y)-\phi(x-y)|}{2} .
$$

Clearly, $\mathscr{H}(\phi)$ is an extension of $\phi$, i.e., $\mathscr{H}(\phi)(x+i 0)=\phi(x)$. Given $\phi \in \operatorname{Homeo}(\mathbf{R})$, for each $z=x+i y$ we have $|z|^{2}=x^{2}+y^{2}=\left((x-y)^{2}+(x+y)^{2}\right) / 2$ and, in particular,

$$
\frac{1}{\sqrt{2}} \max \{|x-y|,|x+y|\} \leq|z| \leq \max \{|x-y|,|x+y|\} \text {. }
$$

Similarly,

$$
|\mathscr{H}(\phi)(z)|^{2}=\frac{1}{2}\left[(\phi(x-y))^{2}+(\phi(x+y))^{2}\right]
$$


and

$$
\frac{1}{\sqrt{2}} \max \{|\phi(x-y)|,|\phi(x+y)|\} \leq|\mathscr{H}(\phi)(z)| \leq \max \{|\phi(x-y)|,|\phi(x+y)|\} .
$$

Since $|\phi(t)| \rightarrow \infty$ for $|t| \rightarrow \infty$, we have $\max \{|\phi(x-y)|,|\phi(x+y)|\} \rightarrow \infty$ for $\max \{|x-y|,|x+y|\} \rightarrow \infty$. We conclude that $\mathscr{H}(\phi)(z) \rightarrow \infty$ for $z \rightarrow \infty$.

Theorem 3.1. The operator $\mathscr{H}$ has the following properties:

(1) $\mathscr{H}\left(\phi_{1} \circ \phi_{2}\right)=\mathscr{H}\left(\phi_{1}\right) \circ \mathscr{H}\left(\phi_{2}\right)$ for all $\phi_{1}, \phi_{2} \in \operatorname{Homeo}(\mathbf{R})$;

(2) $\mathscr{H}(a t+b)=a z+b$ for all $a>0$ and $b \in \mathbf{R}$;

(3) $\mathscr{H}(\phi) \in \operatorname{Homeo}\left(\mathbf{H}^{2}\right)$ for each $\phi \in \operatorname{Homeo}(\mathbf{R})$;

(4) $\mathscr{H}$ is continuous in the topology of uniform convergence on compact sets;

(5) If $\phi$ is quasisymmetric, then $\mathscr{H}(\phi)$ is a quasi-isometry;

(6) If $G$ is a convergence group, then so is $\mathscr{H}(G)$.

Proof. The proofs of properties (1)-(4) are straightforward. We provide them here for completeness. We begin with Property (1). Given $\phi_{1}, \phi_{2} \in \operatorname{Homeo}(\mathbf{R})$, let $\phi=\phi_{1} \circ \phi_{2}$. Observe that every self-homeomorphism of $\mathbf{R}$ is either strictly increasing or strictly decreasing. There are four possibilities.

Case 1: $\phi_{1}$ and $\phi_{2}$ are increasing. Then $\phi$ is increasing and

$$
\begin{aligned}
\mathscr{H}(\phi)(x+i y) & =\frac{\phi(x+y)+\phi(x-y)}{2}+i \frac{\phi(x+y)-\phi(x-y)}{2} \\
& =\frac{\phi_{1}\left(\phi_{2}(x+y)\right)+\phi_{1}\left(\phi_{2}(x-y)\right)}{2}+i \frac{\phi_{1}\left(\phi_{2}(x+y)\right)-\phi_{1}\left(\phi_{2}(x-y)\right)}{2} .
\end{aligned}
$$

On the other hand, we have $u+v=\phi_{2}(x+y)$ and $u-v=\phi_{2}(x-y)$, where

$$
u=\frac{\phi_{2}(x+y)+\phi_{2}(x-y)}{2} \quad \text { and } \quad v=\frac{\phi_{2}(x+y)-\phi_{2}(x-y)}{2} .
$$

Hence

$$
\begin{aligned}
& \mathscr{H}\left(\phi_{1}\right)\left(\mathscr{H}\left(\phi_{2}\right)(x+i y)\right)=\mathscr{H}\left(\phi_{1}\right)(u+i v) \\
& =\frac{\phi_{1}\left(\phi_{2}(x+y)\right)+\phi_{1}\left(\phi_{2}(x-y)\right)}{2}+i \frac{\phi_{1}\left(\phi_{2}(x+y)\right)-\phi_{1}\left(\phi_{2}(x-y)\right)}{2} .
\end{aligned}
$$

Case 2: $\phi_{1}$ is increasing and $\phi_{2}$ is decreasing. Then $\phi$ is decreasing and

$$
\begin{aligned}
\mathscr{H}(\phi)(x+i y) & =\frac{\phi(x+y)+\phi(x-y)}{2}+i \frac{|\phi(x+y)-\phi(x-y)|}{2} \\
& =\frac{\phi_{1}\left(\phi_{2}(x+y)\right)+\phi_{1}\left(\phi_{2}(x-y)\right)}{2}+i \frac{\phi_{1}\left(\phi_{2}(x-y)\right)-\phi_{1}\left(\phi_{2}(x+y)\right)}{2} .
\end{aligned}
$$

On the other hand, we have $u+v=\phi_{2}(x-y)$ and $u-v=\phi_{2}(x+y)$, where

$$
u=\frac{\phi_{2}(x+y)+\phi_{2}(x-y)}{2} \text { and } v=\frac{\phi_{2}(x-y)-\phi_{2}(x+y)}{2} \text {. }
$$

Hence

$$
\begin{aligned}
& \mathscr{H}\left(\phi_{1}\right)\left(\mathscr{H}\left(\phi_{2}\right)(x+i y)\right)=\mathscr{H}\left(\phi_{1}\right)(u+i v) \\
& =\frac{\phi_{1}\left(\phi_{2}(x-y)\right)+\phi_{1}\left(\phi_{2}(x+y)\right)}{2}+i \frac{\phi_{1}\left(\phi_{2}(x-y)\right)-\phi_{1}\left(\phi_{2}(x+y)\right)}{2} .
\end{aligned}
$$

The cases when both $\phi_{1}$ and $\phi_{2}$ are decreasing or when $\phi_{1}$ is decreasing and $\phi_{2}$ is increasing are dealt with in a similar fashion. Hence in all four cases we have $\mathscr{H}(\phi)=\mathscr{H}\left(\phi_{1}\right) \circ \mathscr{H}\left(\phi_{2}\right)$. 
To prove Property (2), we observe that if $\phi(t)=a t+b$ for some $a, b \in \mathbf{R}$ with $a>0$ and if $z=x+i y$, then

$$
\mathscr{H}(\phi)(z)=\frac{a(x+y)+b+a(x-y)+b}{2}+i \frac{|a(x+y)+b-(a(x-y)+b)|}{2}=a z+b .
$$

To prove property $(3)$, we first show that $\mathscr{H}(\phi)$ is bijective. Without loss of generality we can assume that $\phi$ is increasing. Let $\mathscr{H}(\phi)(x+i y)=\mathscr{H}(\phi)(u+i v)$. Then $\phi(x+y)+\phi(x-y)=\phi(u+v)+\phi(u-v)$ and $\phi(x+y)-\phi(x-y)=\phi(u+v)-\phi(u-v)$, which implies $\phi(x+y)=\phi(u+v)$ and $\phi(x-y)=\phi(u-v)$. Hence $x+i y=u+i v$, i.e., $\mathscr{H}(\phi)$ is one-to-one. To show that $\mathscr{H}(\phi)$ is onto, let $u+i v \in \mathbf{H}^{2}$ be arbitrary point. Put

$$
x=\frac{\phi^{-1}(u+v)+\phi^{-1}(u-v)}{2} \quad \text { and } \quad y=\frac{\phi^{-1}(u+v)-\phi^{-1}(u-v)}{2} .
$$

Then $\phi(x+y)=u+v$ and $\phi(x-y)=u-v$ and, consequently

$$
u=\frac{\phi(x+y)+\phi(x-y)}{2} \quad \text { and } \quad v=\frac{\phi(x+y)-\phi(x-y)}{2} .
$$

Hence $\mathscr{H}(\phi)(x+i y)=u+i v$, i.e., $\mathscr{H}(\phi)$ is onto. Next, for each $\phi \in \operatorname{Homeo}(\mathbf{R})$, the continuity of $\mathscr{H}(\phi)$ follows from the construction and since $\mathscr{H}\left(\phi^{-1}\right)=\mathscr{H}(\phi)^{-1}$ we also obtain the continuity of $\mathscr{H}(\phi)^{-1}$. Thus, $\mathscr{H}(\phi) \in \operatorname{Homeo}\left(\mathbf{H}^{2}\right)$.

Next, we prove property (4). Suppose that a sequence $\left\{\phi_{k}\right\}$ converges to $\phi$ uniformly on compact sets. Without loss of generality we may assume that $\phi$ as well as all $\phi_{k}$ 's are increasing homeomorphisms. Let $C \subset \mathbf{C}$ be any compact set. We want to show that $\mathscr{H}\left(\phi_{k}\right)$ converges to $\mathscr{H}(\phi)$ uniformly on $C$. Set

$$
E=\{t \in \mathbf{R}: t=x-y \text { or } t=x+y \text { for some } x+i y \in C\} .
$$

Clearly, $E$ is compact. Let $\varepsilon>0$ be given. Since $\left\{\phi_{k}\right\}$ converges to $\phi$ uniformly on $E$, there exists $N$ such that $\left|\phi_{n}(t)-\phi(t)\right|<\varepsilon / 2$ for all $t \in E$ and all $n \geq N$. Since

$$
\left|\mathscr{H}\left(\phi_{k}\right)(z)-\mathscr{H}(\phi)(z)\right| \leq\left|\phi_{k}(x+y)-\phi(x+y)\right|+\left|\phi_{k}(x-y)-\phi(x-y)\right|,
$$

we have $\left|\mathscr{H}\left(\phi_{n}\right)(z)-\mathscr{H}(\phi)(z)\right|<\varepsilon$ whenever $n \geq N$ and $z=x+i y \in C$, as required.

We prove property (5). Suppose $\phi$ is $\eta$-quasisymmetric. Then $\phi$ is $(\lambda, \alpha)$ quasisymmetric for some $\lambda \geq 1$ and $\alpha \geq 1$ depending only on $\eta$. Let $z=x+i y$ and $w=u+i v$ be arbitrary points in $\mathbf{H}^{2}$. Put $\mathscr{H}(\phi)(z)=z^{\prime}=x^{\prime}+i y^{\prime}$ and $\mathscr{H}(\phi)(w)=w^{\prime}=u^{\prime}+i v^{\prime}$. Without loss of generality we can assume that $\phi$ is increasing. Then $x^{\prime}-y^{\prime}=\phi(x-y), x^{\prime}+y^{\prime}=\phi(x+y), u^{\prime}-v^{\prime}=\phi(u-v)$ and $u^{\prime}+v^{\prime}=\phi(u+v)$. In particular,

$$
\frac{\rho\left(z^{\prime}, w^{\prime}\right)}{2 \operatorname{Im}\left[z^{\prime}\right]}=\frac{|s-t|}{\phi(x+y)-\phi(x-y)} \quad \text { and } \quad \frac{\rho\left(z^{\prime}, w^{\prime}\right)}{2 \operatorname{Im}\left[w^{\prime}\right]}=\frac{|\tilde{s}-\tilde{t}|}{\phi(u+v)-\phi(u-v)}
$$

for some $s, \tilde{s} \in\{\phi(x-y), \phi(x+y)\}$ and $t, \tilde{t} \in\{\phi(u-v), \phi(u+v)\}$. Then using (2.2) and (2.3) we obtain

$$
\begin{aligned}
h\left(z^{\prime}, w^{\prime}\right) & \leq \log \frac{\rho^{2}\left(z^{\prime}, w^{\prime}\right)}{\operatorname{Im}\left[z^{\prime}\right] \operatorname{Im}\left[w^{\prime}\right]}=\log \left(\frac{\rho\left(z^{\prime}, w^{\prime}\right)}{2 \operatorname{Im}\left[z^{\prime}\right]} \frac{\rho\left(z^{\prime}, w^{\prime}\right)}{2 \operatorname{Im}\left[w^{\prime}\right]}\right)+\log 4 \\
& \leq \log \left(\frac{\rho^{2}(z, w)}{\operatorname{Im}[z] \operatorname{Im}[w]}\right)^{\alpha}+\log 4 \lambda^{2} \leq \alpha h(z, w)+\log \left(4^{\alpha+1} \lambda^{2}\right) .
\end{aligned}
$$


Conversely, using the facts that $\phi^{-1}$ is $\left(\lambda^{\alpha}, \alpha\right)$-quasisymmetric and $\mathscr{H}\left(\phi^{-1}\right)=\mathscr{H}(\phi)^{-1}$ we obtain

$$
h(z, w)=h\left(\mathscr{H}\left(\phi^{-1}\right)\left(z^{\prime}\right), \mathscr{H}\left(\phi^{-1}\right)\left(w^{\prime}\right)\right) \leq \alpha h\left(z^{\prime}, w^{\prime}\right)+\log \left(4^{\alpha+1} \lambda^{2 \alpha}\right) .
$$

Hence

$$
h\left(z^{\prime}, w^{\prime}\right) \geq \frac{1}{\alpha} h(z, w)-\log \left(4^{(\alpha+1) / \alpha} \lambda^{2}\right) \geq \frac{1}{\alpha} h(z, w)-\log \left(4^{\alpha+1} \lambda^{2}\right) .
$$

Thus, $\mathscr{H}(\phi)$ is $\left(\alpha, \log \left(4^{\alpha+1} \lambda^{2}\right)\right)$-quasi-isometry.

Finally, we prove (6). Let $G \subset \operatorname{Homeo}(\mathbf{R})$ be a convergence group. Then $G$ is conjugate in $\operatorname{Homeo}(\overline{\mathbf{R}})$ to a Fuchsian group, say $G=f \circ \tilde{H} \circ f^{-1}$, by a well-known result due to Casson and Jungreis [4] and Gabai [9]. Recall that Fuchsian groups are convergence groups. If $f^{-1}(\infty)=\infty$, we let $\mu_{f}$ be the identity map. Otherwise, we let $\mu_{f}$ be an inversion about the point $f^{-1}(\infty)$. That is, $\mu_{f}(t)=1 /(t-a)+a$, where $a=f^{-1}(\infty)$. Then $G=\phi \circ H \circ \phi^{-1}$, where $\phi=f \circ \mu_{f}, H=\mu_{f} \circ \tilde{H} \circ \mu_{f}$. Note that $\phi \in \operatorname{Homeo}(\mathbf{R})$ and that $H$ is a Fuchsian group whose elements fix $\infty$. Thus, $\mathscr{H}(G)=\mathscr{H}(\phi) \circ H \circ \mathscr{H}(\phi)^{-1}$ and hence it is a convergence group.

Next, we will show that the extensions $\mathscr{H}(\phi)$ of quasisymmetric mappings $\phi$ are, in fact, stronger than quasi-isometry. Namely, they are biuniform. This would provide an alternative proof of Theorem 3.1(5), but here one has no control of the constants of quasiisometry since the proof is based on a compactness argument.

Lemma 3.2. If $\phi: \mathbf{R} \rightarrow \mathbf{R}$ is $K$-quasisymmetric, then $\mathscr{H}(\phi)$ is $\left(\eta, \eta^{\prime}\right)$-biuniform, where $\eta$ and $\eta^{\prime}$ depend only on $K$.

Proof. Let $\mathscr{F}_{K}$ be the family of $K$-quasisymmetric mappings of $\mathbf{R}$ normalized at 0 and 1. That is, $\phi(0)=0$ and $\phi(1)=1$ for each $\phi \in \mathscr{F}_{K}$. This family is compact, i.e., every sequence has a subsequence converging uniformly on compact subsets (see, [3, Theorem 2] or [1, p. 41]). Let $\mathscr{G}_{K}=\left\{\mathscr{H}(\phi): \phi \in \mathscr{F}_{k}\right\}$. Using continuity of $\mathscr{H}$ one easily shows that $\mathscr{G}_{K}$ is equicontinuous at any point of the hyperbolic space $\mathbf{H}^{2}$, say at $i$. In particular, the function $\zeta:[0, \infty) \rightarrow[0, \infty)$, defined by

$$
\zeta(r)=\sup _{\Phi \in \mathscr{G}_{K}} \max _{h(z, i)=r} h(\Phi(z), \Phi(i)),
$$

is bounded on bounded sets, $\zeta(r) \rightarrow 0$ as $r \rightarrow 0$ and

$$
h(\Phi(z), \Phi(i)) \leq \zeta(h(z, i))
$$

for all $\Phi \in \mathscr{G}_{K}$ and for all $z \in \mathbf{H}^{2}$. Clearly, $\zeta$ depends only on $K$. Then there exists a homeomorphism $\eta:[0, \infty) \rightarrow[0, \infty)$, which depends only on $\zeta$, such that $\zeta(t) \leq \eta(t)$ for all $t \geq 0$. Thus,

$$
h(\mathscr{H}(\phi)(z), \mathscr{H}(\phi)(i)) \leq \eta(h(z, i))
$$

for all $\phi \in \mathscr{F}_{K}$ and for all $z \in \mathbf{H}^{2}$.

Suppose now that $\phi$ is an arbitrary $K$-quasisymmetric mapping of $\mathbf{R}$, not necessarily normalized. Let $z_{1}, z_{2} \in \mathbf{H}^{2}$ be arbitrary points. Without loss of generality we can assume that $\phi$ is increasing. Let $\sigma$ and $\tau$ be similarities of $\mathbf{R}$ such that $\sigma \circ \phi \circ \tau$ is in $\mathscr{F}_{K}, \mathscr{H}(\tau)(i)=z_{1}$ and $\mathscr{H}(\tau)(z)=z_{1}$ for some $z \in \mathbf{H}^{2}$. Then

$$
h\left(\mathscr{H}(\phi)\left(z_{1}\right), \mathscr{H}(\phi)\left(z_{2}\right)\right)=h(\mathscr{H}(\sigma \circ \phi \circ \tau)(z), \mathscr{H}(\sigma \circ \phi \circ \tau)(i)) \leq \eta(h(z, i)),
$$

completing the proof of the second inequality. 
To prove the first inequality, we observe that the map $\phi^{-1}$ is $K^{\prime}$-quasisymmetric with $K^{\prime}$ depending only on $K$. Hence there exists a homeomorphism $\xi$ of $[0, \infty)$ depending only on $K$ such that

$$
h\left(\mathscr{H}\left(\phi^{-1}\right)\left(w_{1}\right), \mathscr{H}\left(\phi^{-1}\right)\left(w_{2}\right)\right) \leq \xi\left(h\left(w_{1}, w_{2}\right)\right) \text { for all } w_{1}, w_{2} \in \mathbf{H}^{2} .
$$

By choosing $w_{1}=\mathscr{H}(\phi)\left(z_{1}\right), w_{2}=\mathscr{H}(\phi)\left(z_{2}\right)$ and $\eta^{\prime}=\xi^{-1}$, we see that

$$
\eta^{\prime}\left(h\left(z_{1}, z_{2}\right)\right) \leq h\left(\mathscr{H}(\phi)\left(z_{1}\right), \mathscr{H}(\phi)\left(z_{2}\right)\right)
$$

for all $z_{1}, z_{2} \in \mathbf{H}^{2}$, completing the proof.

We end this section with a construction of an extension operator $\mathscr{H}_{o}$ from the group Homeo ${ }_{+}\left(\mathbf{S}^{1}\right)$ of all orientation preserving homeomorphisms of $\mathbf{S}^{1}$ into the group Homeo $\left(\mathbf{D}^{2}\right)$ compatible with composition. The operator $\mathscr{H}_{o}$ will have a property that $\mathscr{H}_{o}(f)(0)=0$ for each $f \in \mathrm{Homeo}_{+}\left(\mathbf{S}^{1}\right)$. Let $T: \mathbf{C} \rightarrow \mathbf{C}$ be a translation given by $T(z)=z+1$. We also denote by $T$ the restriction of $T$ to $\mathbf{H}^{2}$ as well as to $\mathbf{R}$. Note that $\mathscr{H}$ commutes with $T$, i.e., $\mathscr{H}(f+1)=\mathscr{H}(f)+1$ for all $f \in \operatorname{Homeo}(\mathbf{R})$. Let $M$ be the quotient space of $\mathbf{H}^{2}$ by the cyclic group generated by $T$, i.e., $M=\mathbf{H}^{2} /\langle T\rangle$. Then the map $\mu: M \rightarrow \mathbf{D}^{2} \backslash\{0\}$, given by

$$
\mu(<z>)=\frac{1}{1+\operatorname{Im}[z]} e^{i 2 \pi \operatorname{Re}[z]},
$$

is a homeomorphism. In particular, the map $f \mapsto \mu \circ f \circ \mu^{-1}$ establishes an isomorphism between the group Homeo $(M)$ and a subgroup $\left\{f \in \operatorname{Homeo}\left(\mathbf{D}^{2}\right): f(0)=0\right\}$ of the group Homeo $\left(\mathbf{D}^{2}\right)$.

Next, let $\operatorname{Homeo}^{\mathrm{T}}(\mathbf{R})$ be the centralizer of $T$ in $\operatorname{Homeo}(\mathbf{R})$, i.e., $\operatorname{Homeo}^{\mathrm{T}}(\mathbf{R})=$ $\{f \in \operatorname{Homeo}(\mathbf{R}): f \circ T=T \circ f\}$. Given $f \in \mathrm{Homeo}_{+}\left(\mathbf{S}^{1}\right)$, we define

$$
\Theta(f):[0,1) \rightarrow\left[\frac{\arg (f(1))}{2 \pi}, \frac{\arg (f(1))}{2 \pi}+1\right) .
$$

by the relation $e^{i 2 \pi \Theta(f)(t)}=f\left(e^{i 2 \pi t}\right)$. We then extend $\Theta(f)$ to all $\mathbf{R}$, also denoted by $\Theta(f)$, in a unique manner using the relation $\Theta(f)(t+1)=\Theta(f)(t)+1$. Hence $\Theta(f) \in$ Homeo $^{\mathrm{T}}(\mathbf{R})$. Observe that $\Theta: \mathrm{Homeo}_{+}\left(\mathbf{S}^{1}\right) \rightarrow \operatorname{Homeo}^{\mathrm{T}}(\mathbf{R})$ is a homomorphism. Indeed, it is enough to show that $\Theta(f \circ g)(t)=(\theta(f) \circ \Theta(g))(t)$ for all $f, g \in$ Homeo $_{+}\left(\mathbf{S}^{1}\right)$ and for each $t \in[0,1)$. We have

$$
e^{i 2 \pi \Theta(f \circ g)(t)}=(f \circ g)\left(e^{i 2 \pi t}\right)=f\left(g\left(e^{i 2 \pi t}\right)\right)=f\left(e^{i 2 \pi \Theta(g)(t)}\right)=e^{i 2 \pi(\Theta(f) \circ \Theta(g))(t)} .
$$

Since

$$
\Theta(f \circ g)(t),(\Theta(f) \circ \Theta(g))(t) \in\left[\frac{\arg (f(g(1)))}{2 \pi}, \frac{\arg (f(g(1)))}{2 \pi}+1\right),
$$

we obtain $\Theta(f \circ g)(t)=(\theta(f) \circ \Theta(g))(t)$, as required. Observe that for each $f \in \operatorname{Homeo}^{\mathrm{T}}(\mathbf{R})$ the extension $\mathscr{H}(f)$ acts on $M$ as an orientation preserving homeomorphism. We now define $\mathscr{H}_{o}: \operatorname{Homeo}_{+}\left(\mathbf{S}^{1}\right) \rightarrow \operatorname{Homeo}\left(\mathbf{D}^{2}\right)$ by

$$
\mathscr{H}_{o}(f)=\mu \circ \mathscr{H}(\Theta(f)) \circ \mu^{-1} .
$$

More precisely, $\mathscr{H}_{o}(f)(z)=\mu\left(<\mathscr{H}(\Theta(f))\left(\mu^{-1}(z)\right)>\right)$ if $z \neq 0$ and we define $\mathscr{H}_{o}(f)(0)=0$ by continuity since for each $\phi \in \operatorname{Homeo}(\mathbf{R})$, we have $\mathscr{H}(\phi)(z) \rightarrow \infty$ as $z \rightarrow \infty$. Note that the operator $\mathscr{H}_{o}$ can be extended to all of Homeo $\left(\mathbf{S}^{1}\right)$ by setting $\mathscr{H}_{o}(f)=\overline{\mathscr{H}_{o}(\bar{f})}$ for each orientation reversing homeomorphism $f$ of $\mathbf{S}^{1}$. 


\section{The Beurling-Ahlfors extension}

In this section we show that the extension operator $\mathscr{H}$ lies within a bounded hyperbolic distance of the Beurling-Ahlfors extension when restricted to quasisymmetric mappings. Combining this result with the composition property of $\mathscr{H}$ we then show that the Beurling-Ahlfors extension is a "rough" homomorphism.

To confirm with the original setting of Beurling and Ahlfors, we assume throughout this section that all quasisymmetric mappings are strictly increasing. Recall that given a $K$-quasisymmetric mapping $\phi: \mathbf{R} \rightarrow \mathbf{R}$, the extension $\mathscr{B}(\phi): \mathbf{H}^{2} \rightarrow \mathbf{H}^{2}$ is defined by

$$
\mathscr{B}(\phi)(z)=\mathscr{B}(\phi)(x+i y)=u(x, y)+i v(x, y)
$$

where

$$
u(x, y)=\frac{1}{2} \int_{0}^{1}[\phi(x+t y)+\phi(x-t y)] d t
$$

and

$$
v(x, y)=\frac{1}{2} \int_{0}^{1}[\phi(x+t y)-\phi(x-t y)] d t .
$$

The mapping $\mathscr{B}(\phi)$ is $2 K(K+1)$-quasiconformal. Moreover, it is $4 K^{2}(K+1)$ biLipschitz in the hyperbolic metric $([1])$. Note that in their original construction, Beurling and Ahlfors multiplied the imaginary part $v(x, y)$ by an appropriate constant (depending only on $K$ ) in order to achieve $K^{2}$-quasiconformality ([3]). One easily verifies that $\mathscr{B}(\sigma \circ \phi \circ \tau)=\sigma \circ \mathscr{B}(\phi) \circ \tau$, where $\sigma(z)=a z+b$ and $\tau(z)=c z+d$ are conformal maps of the upper-half plane $\mathbf{H}^{2}$ onto itself. Notice that $\mathscr{B}(a t+b) \neq a z+b$ for any $a, b \in \mathbf{R}, a>0$.

Theorem 4.1. For each strictly increasing $K$-quasisymmetric mapping $\phi$ we have

$$
h(\mathscr{H}(\phi)(z), \mathscr{B}(\phi)(z)) \leq \mu(K) \quad \text { for all } \quad z \in \mathbf{H}^{2}
$$

where

$$
\mu(K)=2 \log \frac{8 K^{3}+12 K^{2}-1}{2 K+1} .
$$

Proof. First, we assume that $\phi$ is normalized, i.e., $\phi(0)=0$ and $\phi(1)=1$, and show that $h(\mathscr{H}(\phi)(i), \mathscr{B}(\phi)(i)) \leq \mu(K)$. We have

$$
\operatorname{Re}[\mathscr{H}(\phi)(i)]=\frac{1}{2}[\phi(-1)+\phi(1)]=\frac{1}{2}(1+\phi(-1))
$$

and

Similarly,

$$
\operatorname{Im}[\mathscr{H}(\phi)(i)]=\frac{1}{2}[\phi(1)-\phi(-1)]=\frac{1}{2}(1-\phi(-1)) .
$$

$$
\operatorname{Re}[\mathscr{B}(\phi)(i)]=\frac{1}{2} \int_{0}^{1}[\phi(t)+\phi(-t)] d t=\frac{1}{2}\left[\int_{0}^{1} \phi(t) d t+\int_{-1}^{0} \phi(t) d t\right]
$$

and

$$
\operatorname{Im}[\mathscr{B}(\phi)(i)]=\frac{1}{2} \int_{0}^{1}[\phi(t)-\phi(-t)] d t=\frac{1}{2}\left[\int_{0}^{1} \phi(t) d t-\int_{-1}^{0} \phi(t) d t\right] .
$$


An easy computation using (2.4) yields

$$
-K \leq \phi(-1) \leq \frac{-1}{K}, \quad \frac{-K^{2}}{K+1} \leq \phi(-1 / 2) \leq \frac{-1}{K(K+1)}
$$

and

$$
\frac{1}{K+1} \leq \phi(1 / 2) \leq \frac{K}{K+1}
$$

According to Lemma 3 [1] (see, also [3, (19)]) we have

$$
\frac{1}{K+1} \leq \int_{0}^{1} \phi(t) d t \leq \frac{K}{K+1}
$$

We also have

$$
-\frac{K(2 K+1)}{2(K+1)} \leq \frac{\phi(-1 / 2)+\phi(-1)}{2} \leq \int_{-1}^{0} \phi(t) d t \leq \frac{\phi(-1 / 2)}{2} \leq-\frac{1}{2 K(K+1)} .
$$

Combining these estimates we obtain

$$
a \leq \operatorname{Re}[\mathscr{H}(\phi)(i)] \leq a^{\prime} \quad \text { and } \quad b \leq \operatorname{Im}[\mathscr{H}(\phi)(i)] \leq b^{\prime}
$$

where

$$
a=-\frac{K-1}{2}, \quad a^{\prime}=\frac{K-1}{2 K}, \quad b=\frac{K+1}{2 K}, \quad b^{\prime}=\frac{K+1}{2} .
$$

Similarly,

$$
c \leq \operatorname{Re}[\mathscr{B}(\phi)(i)] \leq c^{\prime} \quad \text { and } \quad d \leq \operatorname{Im}[\mathscr{B}(\phi)(i)] \leq d^{\prime}
$$

where

$$
c=-\frac{2 K^{2}+K-2}{2(K+1)}, \quad c^{\prime}=\frac{2 K^{2}-1}{4 K(K+1)}, \quad d=\frac{2 K+1}{4 K(K+1)}, \quad d^{\prime}=\frac{2 K^{2}+3 K}{4(K+1)} .
$$

Observe that $d \leq b, d^{\prime} \leq b^{\prime}$ and $c \leq a \leq a^{\prime} \leq c^{\prime}$. It follows that

$$
\rho(\mathscr{H}(\phi)(i), \mathscr{B}(\phi)(i)) \leq c^{\prime}-c+2 b^{\prime}=\frac{8 K^{3}+12 K^{2}-1}{4 K(K+1)}
$$

and using Lemma 2.3 we obtain

$$
h(\mathscr{H}(\phi)(i), \mathscr{B}(\phi)(i)) \leq \log \frac{\rho^{2}(\mathscr{H}(\phi)(i), \mathscr{B}(\phi)(i))}{\operatorname{Im}[\mathscr{H}(\phi)(i)] \operatorname{Im}[\mathscr{B}(\phi)(i)]} \leq 2 \log \frac{8 K^{3}+12 K^{2}-1}{2 K+1}
$$

as required.

Finally, for arbitrary $\phi$ and $z$ we let $\sigma$ and $\tau$ be similarities so that $z=\tau(i)$ and that $\sigma \circ \phi \circ \tau$ is normalized. Then

$$
\begin{aligned}
h(\mathscr{H}(\phi)(z), \mathscr{B}(\phi)(z)) & =h(\mathscr{H}(\phi) \circ \tau(i), \mathscr{B}(\phi) \circ \tau(i)) \\
& =h(\mathscr{H}(\phi \circ \tau)(i), \mathscr{B}(\phi \circ \tau)(i)) \\
& =h(\sigma \circ \mathscr{H}(\phi \circ \tau)(i), \sigma \circ \mathscr{B}(\phi \circ \tau)(i)) \\
& =h(\mathscr{H}(\sigma \circ \phi \circ \tau)(i), \mathscr{B}(\sigma \circ \phi \circ \tau)(i)) \leq \mu(K),
\end{aligned}
$$

completing the proof.

As both $\mathscr{H}(\phi)$ and $\mathscr{B}(\phi)$ are quasi-isometric extensions of $\phi$, it follows from a result of Tukia ([16, Theorem 3I]) that there exists a constant $C$ depending only on $K$ such that $h(\mathscr{H}(\phi)(z), \mathscr{B}(\phi)(z)) \leq C$. However, since the proof of Theorem 3I is 
based on a compactness argument, it does not yield an explicit constant. Combining Theorem 4.1 with the composition property of $\mathscr{H}$ we obtain the following corollary.

Corollary 4.2. There is a constant $D$ depending only on $K$ such that if $\phi$ and $\psi$ are $K$-quasisymmetric, then

$$
h(\mathscr{B}(\psi \circ \phi)(z), \mathscr{B}(\psi) \circ \mathscr{B}(\phi)(z)) \leq D
$$

for every $z \in \mathbf{H}^{2}$.

Proof. Let $\phi$ and $\psi$ be $K$-quasisymmetric mappings and let $z \in \mathbf{H}^{2}$ be arbitrary point. Note that the map $\psi \circ \phi$ is $K^{\prime}$-quasisymmetric for $K^{\prime}$ depending only on $K$. Using the composition property of $\mathscr{H}$ and the triangle inequality we have

$$
\begin{aligned}
h(\mathscr{B}(\psi \circ \phi)(z), \mathscr{B}(\psi) \circ \mathscr{B}(\phi)(z)) \leq & h(\mathscr{H}(\psi \circ \phi)(z), \mathscr{B}(\psi \circ \phi)(z)) \\
& +h(\mathscr{H}(\psi) \circ \mathscr{H}(\phi)(z), \mathscr{B}(\psi) \circ \mathscr{B}(\phi)(z)) .
\end{aligned}
$$

Put $z_{1}=\mathscr{H}(\phi)(z)$ and $z_{2}=\mathscr{B}(\phi)(z)$. Then

$$
h\left(\mathscr{H}(\psi)\left(z_{1}\right), \mathscr{B}(\psi)\left(z_{2}\right)\right) \leq h\left(\mathscr{H}(\psi)\left(z_{1}\right), \mathscr{B}(\psi)\left(z_{1}\right)\right)+h\left(\mathscr{B}(\psi)\left(z_{1}\right), \mathscr{B}(\psi)\left(z_{2}\right)\right) .
$$

Since $\mathscr{B}(\psi)$ is $4 K^{2}(K+1)$-biLipscitz in the hyperbolic metric, we obtain

$$
h\left(\mathscr{B}(\psi)\left(z_{1}\right), \mathscr{B}(\psi)\left(z_{2}\right)\right) \leq 4 K^{2}(K+1) h(\mathscr{H}(\phi)(z), \mathscr{B}(\phi)(z)) .
$$

Using Theorem 4.1 we have

$$
\begin{aligned}
h(\mathscr{H}(\psi \circ \phi)(z), \mathscr{B}(\psi \circ \phi)(z)) & \leq \mu\left(K^{\prime}\right), \\
h\left(\mathscr{H}(\psi)\left(z_{1}\right), \mathscr{B}(\psi)\left(z_{1}\right)\right) & \leq \mu(K)
\end{aligned}
$$

and

By combining all these estimates we obtain

$$
h(\mathscr{H}(\phi)(z), \mathscr{B}(\phi)(z)) \leq \mu(K)
$$

$$
h(\mathscr{B}(\psi \circ \phi)(z), \mathscr{B}(\psi) \circ \mathscr{B}(\phi)(z)) \leq D,
$$

where $D=\mu\left(K^{\prime}\right)+\mu(K)\left(4 K^{3}+4 K^{2}+1\right)$.

\section{References}

[1] Ahlfors, L. V.: Lectures on quasiconformal mappings. - Univ. Lecture Ser., AMS, Providence, RI, 2006.

[2] Beardon, A. F.: Geometry of discrete groups. - Grad. Text in Math. 91, New York, SpringerVerlag, 1995.

[3] Beurling, A., and L. V. Ahlfors: The boundary correspondence under quasiconformal mappings. - Acta Math. 96, 1956, 125-142.

[4] Casson, A., and D. Jungreis: Convergence groups and Seifert fibered 3-manifolds. - Invent. Math. 118, 1994, 441-456.

[5] Doundy, A., and C. EARLE: Conformally natural extension of homeomorphisms of the circle. - Acta Math. 157, 1986, 23-48.

[6] Efremovich, V.A., and E. S. Tihomirova: Equimorphisms of hyperbolic spaces. - Izv. Akad. Nauk SSSR Ser. Mat. 28, 1964, 1139-1144 (in Russian). 
[7] Epstein, D. B. A., A. Marden, and V. Markovic: Quasiconformal homeomorphisms and the convex hull boundary. - Ann. of Math. (2) 159, 2004, 305-336.

[8] Epstein, D. B. A., and V. Markovic: Extending homeomorphisms of the circle to quasiconformal homeomorphisms of the disk. - Geom. Topol. 11, 2007, 517-595.

[9] Gabai, D.: Convergence groups are Fuchsian groups. - Ann. of Math. (2) 136, 1992, 447-510.

[10] Gehring, F. W., and G. Martin: Discrete quasiconformal groups I. - Proc. London Math. Soc. 55, 1987, 331-358.

[11] Hinkkanen, A.: Abelian and nondiscrete convergence groups on the circle. - Trans. Amer. Math. Soc. 318, 1990, 87-121.

[12] Martin, G., and P. Tukia: Convergence and Möbius groups. - In: Holomorphic Functions and Moduli II, M.S.R.I. Publications 11, 1988, 113-140.

[13] Norton, A., and D. Sullivan: Wandering domains and invariant conformal structures for mappings of the 2-torus. - Ann. Acad. Sci. Fenn. Ser. A I Math. 21, 1996, 51-68.

[14] Tukia, P.: Quasiconformal extension of quasisymmetric mappings compatible with a Möbius group. - Acta Math. 154, 1985, 153-193.

[15] Tukia, P.: Homeomorphic conjugates of Fuchsian groups. - J. Reine Angew. Math. 391, 1988, $1-54$.

[16] TUkiA, P.: Convergence groups and Gromov's metric hyperbolic spaces. - New Zealand J. Math. 23:2, 1994, 157-187.

[17] Tukia, P., and J. VÄIsÄLÄ: Quasisymmetric embeddings of metric spaces. - Ann. Acad. Sci. Fenn. Ser. A I Math. 5, 1980, 97-114.

[18] VÄısÄLÄ, J.: Gromov hyperbolic spaces. - Expo. Math. 23, 2005, 187-231.

Received 11 February 2009 